\title{
DIFFERENCES BETWEEN MALE AND FEMALE GROWTH AND SEXUAL MATURATION IN TILAPIA (OREOCHROMIS MOSSAMBICUS)
}

\author{
Sandip Bhatta, Toshiharu Iwai, Takeshi Miura, Masato Higuchi, Gersende Maugars, Chiemi \\ Miura* \\ Research Group for Fish Reproductive Physiology, South Ehime Fisheries Research Center, \\ Ehime University, 1289-1 Funakoshi, Ainan, 798-4292, Ehime, Japan \\ *Corresponding author: chiemi8@agr.ehime-u.ac.jp \\ Received 28 March, 2012; Revised 03 June, 2012
}

\begin{abstract}
In tilapia, growth during critical periods of the life cycle varies at different ages of development and is influenced by sexual maturation. The mechanisms controlling gametogenesis and how growth affects this process are poorly understood. This study indicates that tilapia exhibit a sexually dimorphic growth pattern in which males grow faster and bigger than females. During critical periods of development growth patterns of tilapia vary between males and females as they increase in age and sexual maturation. In this study interactions between growth and reproductive development were examined by monitoring and comparing growth rates and reproductive histology. Female growth rate peaked at the very early stages of reproductive development during the cortical alveolar stages and male growth rate peaked after complete sexual maturation during spermiogenesis. The accumulation of cortical alveoli in the oocyte in females and spermiogenesis in males was determined to be the critical developmental periods of tilapia effecting growth rates.
\end{abstract}

Keywords: Gemetogenesis, Growth rate, Meiosis, Oogenesis, Spermatogenesis

\section{INTRODUCTION}

Since male grow faster and bigger than females in most tilapiine species, it is important to determine to what extent these dimorphic characters reflect true dimorphism [1]. However detailed information on why males are bigger than females is lacking. This question becomes especially relevant when considering the fact that sexual maturation may be related at various size and development. Puberty is the developmental period comprising the transition from an immature juvenile to a mature adult state of the reproductive system, i.e. the stage of development during which an individual becomes capable of reproducing sexually, it is characterized by an activation of the brain-pituitary gonadal (BPG) axis. In vertebrates, including nonmammals, germ cell development and maturation appear in a similar fashion [2]. Spermatogenesis in vertebrates can be divided distinct sequential phases: mitotic stem cell renewal, mitotic proliferation of spermatogonia and supporting cells (e.g., Sertoli and Leydig cells), meiosis (the meiotic division of germ cells), and spermiogenesis (the maturation of germ cells into fully functional spermatozoa) $[3,4,5,6]$. In oogenesis, oogonia proliferate by mitosis and subsequently develop into primary oocytes that have initiated meiosis. In general, during oogenesis, primary oocytes arrest division at the diplotene stage in the prophase of the first meiotic division and accumulate yolk during meiotic arrest. Thereafter, oocytes resume the first meiotic division and differentiate into mature eggs through final maturation $[7,8,9,10,11]$. The gonadal development stages synchronize with body size and gonadosomatic index (GSI). Salmon studies have shown that the initial growth rate is a critical period which determines whether animals will undergo gonadal maturation and initiation of spermatogenesis and secondary 
ovarian follicle growth $[12,13]$. However, the physiological significances of these interactions have not been studied much in other fish species. Specifically it is not known if species-specific difference exist and the mechanism by which reproductive maturation influences growth rate to ultimately regulate body size.

In general, there is a relationship between growth and reproduction [13]. The time of sexually maturity which leads to significant changes in body composition, also plays a key role in the growth regulation between both sexes. For a detailed analysis of interaction between growth and reproductive maturation, we utilize Mossambicus tilapia (Oreochromis mossambicus) as a model animal for the study of growth and reproductive physiology, since tilapia have high growth rates, change reproductive cycle within a few days and reach sexual mature until 90 days. The main focus of this study is the differences and interrelationship between growth, growth rate and reproductive maturation in both male and female tilapia.

\section{MATERIALS AND METHODS Animal culture condition}

This study was carried out in accordance with Guideline for animal Care and Use of laboratory Animals in South Ehime Fisheries Research Center, Ehime University. Tilapias (Oreochromis mossambicus) juveniles were obtained from a stock maintained at the laboratory, and fish were maintained in 500 liters tank in a recirculation fresh water system at $28^{\circ} \mathrm{C}$ with UV filtration under a 14L: 10D photoperiod. Fish larvae of both sexes at different developmental stages, i.e, 15 to 120 days after fertilization (daf) were used to this experiment to clarify the differences between male and female growth and reproductive maturation between male and female tilapia.

\section{Fish growth}

The fish body weight $(\mathrm{g})$ and length $(\mathrm{mm})$ were measured at various time points serially between at 15 day time intervals each. Growth differentiation between male and female were analyzed by the simple logistic regression analysis in body weight (g) by using the following simple equations $B W=a / 1+e^{(b-c t) .}$ Where the variables are male: $a=61.127, b=5.1142, c=0.062238$, $\mathrm{R}^{2}=0.999$ and female: $\mathrm{a}=31.599, \mathrm{~b}=4.8172, \mathrm{c}=0.0745, \mathrm{R}^{2}=0.997, \mathrm{t}=$ time and logarithm ' $\mathrm{e}$ ' is irrational constant approximately $2.7118828 \ldots .$. The Gompertz curve fit analysis was derived from the Kaleida graphs, (synergy software, Japan). Gonadosomatic index (GSI) was calculated by gonads weight (g) / body weight (g) X 100 as previously described [13].

\section{Histology}

Histology at various time points was used to determine the germ cells growth and development in the real time. The fragment of the gonadal tissue was fixed in Bouin's solution (water saturated picric acid (71.428\%), formalin (23.809\%) and acetic acid (4.761\%)) for overnight at $4^{\circ} \mathrm{C}$ with shaking. Fixed samples were dehydrated through ethanol and embedded in paraplast, sectioned $(5 \mu \mathrm{m})$, and stained with hematoxylin and eosin. Stages of spermatogenesis and oogenesis were determined by light microscopy (Olympus BX-51) with various magnifications.

\section{Statistical analysis}

All results presented in this study are expressed as the mean \pm SEM. All values were analyzed by the 1-way analysis of variance followed by Bonferroni multicomparison test was used to analyze 
the difference of means using Kaleida Graphs statistical software $(\mathrm{p}<0.05)$ was considered statistically significant.

\section{RESULTS}

Growth differentiation between male and female and relationship among body weight, length, growth rates and gonadosomatic index (GSI)

A
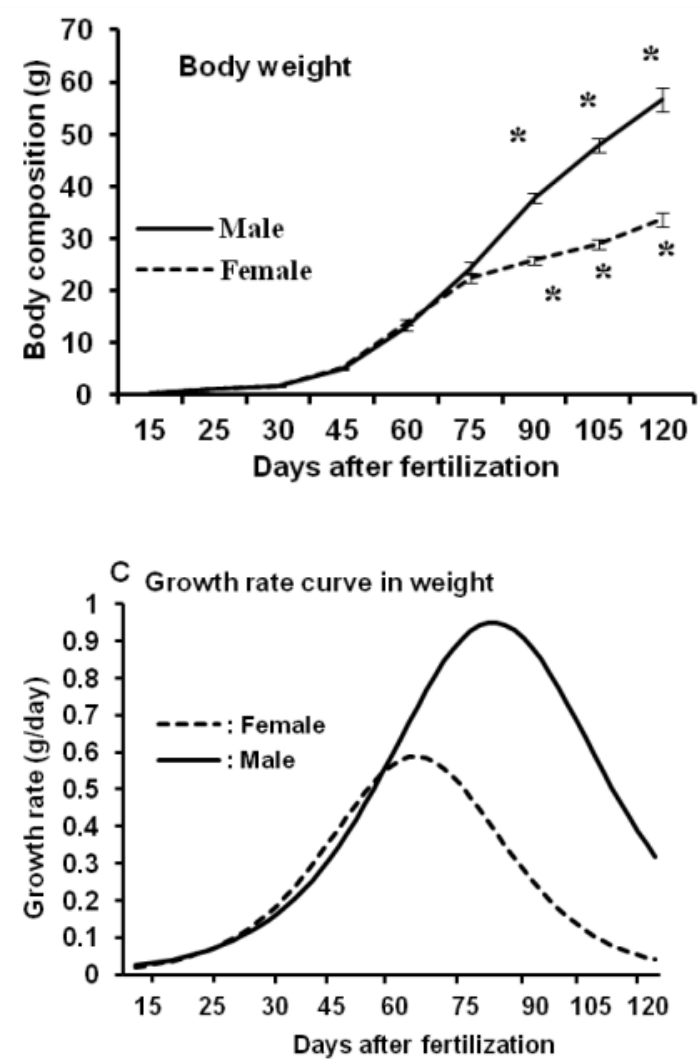
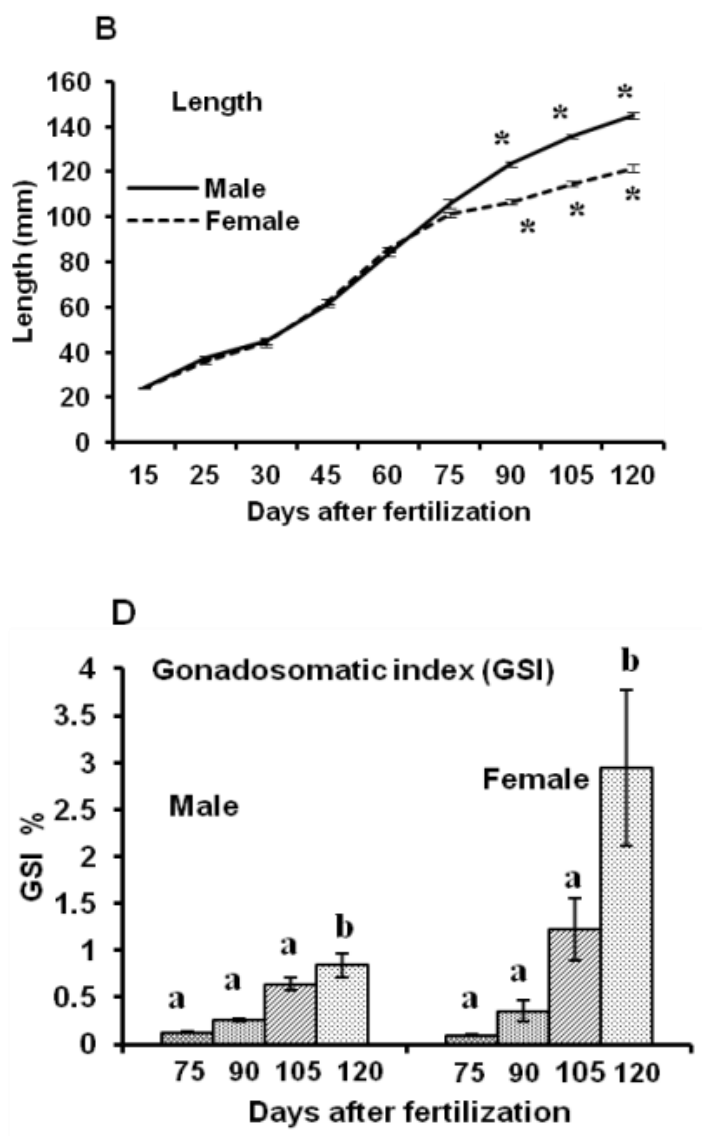

Fig.1 Growth differences between male and female tilapia. (A) Growth in body weight (g). (B) Growth in total length (mm). (C) Growth rates in body weight (g/day) and (D) Gonadosomatic index (GSI) compared between male and female over time. Results are given as means \pm SEM calculated with each groups $(\mathrm{n}=10)$. Asterisks and different letters indicate statistically significant differences between groups. ${ }^{*}$ : $\mathrm{p}<0.0001$, a: $\mathrm{p}<0.05$, b: $\mathrm{p}<0.0001$.

To investigate the state of growth differentiation between males and females; we analyzed difference between male and female fish from 15 days after fertilization (daf) to 120daf in between males and females. The initial male and female total length (TL) $=24.004 \pm 0.28 \mathrm{~mm}$, body weight $(\mathrm{BW})=0.28 \pm 0.009 \mathrm{~g}$, final male $\mathrm{TL}=144.82 \pm 1.51 \mathrm{~mm}, \mathrm{BW}=56.7 \pm 2.29 \mathrm{~g}$, and final female $\mathrm{TL}=121.67 \pm 1.64 \mathrm{~mm}, \mathrm{BW}=33.63 \pm 1.26 \mathrm{~g}$ were recorded (Fig. 1A \& $\mathrm{B}$ ). After 90 daf male and female growth had significant differences $(\mathrm{P}<0.0001)$ in body weight and total length. Males were $1.68^{\mathrm{BW}}$ and $1.19^{\mathrm{TL}}$ fold greater than females at the end of the experiment at 
120 daf (Fig. 1A \& B). The maximum mean growth rate in body weight was observed during 80$90 \mathrm{daf}(0.93 \pm 0.006 \mathrm{~g} / \mathrm{d})$ in males, and 50-60 daf $(0.57 \pm 0.003 \mathrm{~g} / \mathrm{d})$ in female. GSI represent the maturing gonadal proportions of animals which relatively depended on body weight (Fig.1A, vs.1D). The growth pattern of body weight, length and GSI are in sequentially order (compare Fig. 1A, B vs. D). Female GSI significantly increased during spawning reached around 3\% of body weight, and male around $1 \%(\mathrm{p}<0.0001)$ at 120 daf.

\section{Testicular development during spermatogenesis to spermiogenesis is associated with growth rate and body weight}

To investigate the relationship between growth and testicular germ cells, we examined testicular development over time. In sexually immature tilapia undifferentiated testes containing primordial germ cells (PGCs) were observed during 15-25 daf (Fig. 2A, B). With the onset of spermatogenesis, stem cells undergo proliferation of spermatogonia, spermatogonial self-renewal to spermatogonial proliferation through a number of mitotic cells divisions during 30-60 daf (Fig. 2 C, D, E). Type A and type B spermatogonia were located on the edge of lobules and a part of their Sertoli cells were attached to the basement membrane. Concomitantly, multiplication of germ cell takes place, and this development is paralleled by elongation of testicular lamellae and rapid progress of spermatogonial proliferation and entranced into first mitotic division (spermatocysts) around 60 daf (Fig. 2E). After initiating meiosis, cells rapidly underwent to mature sperm production, asynchronously all forms of spermatogenic cells were presented in around 75 daf, spermatogonia (GA \& GB), spermatocyst (SC), spermatids (ST) and spermatozoa (SZ) (Fig. 2F). The spermatids then processed through a morphological metamorphosis during spermiogenesis to form spermatozoa. The expansion of the efferent duct and milt could be acquired by hand-stripping at 90-120 daf indicating full gonad maturation (Fig. $2 \mathrm{G}, \mathrm{H}, \mathrm{I})$. Results show that gonadal development is positively correlated with body weight and GSI. And growth rate was decreased from the initiation of spermiogenesis at 80-90 daf.

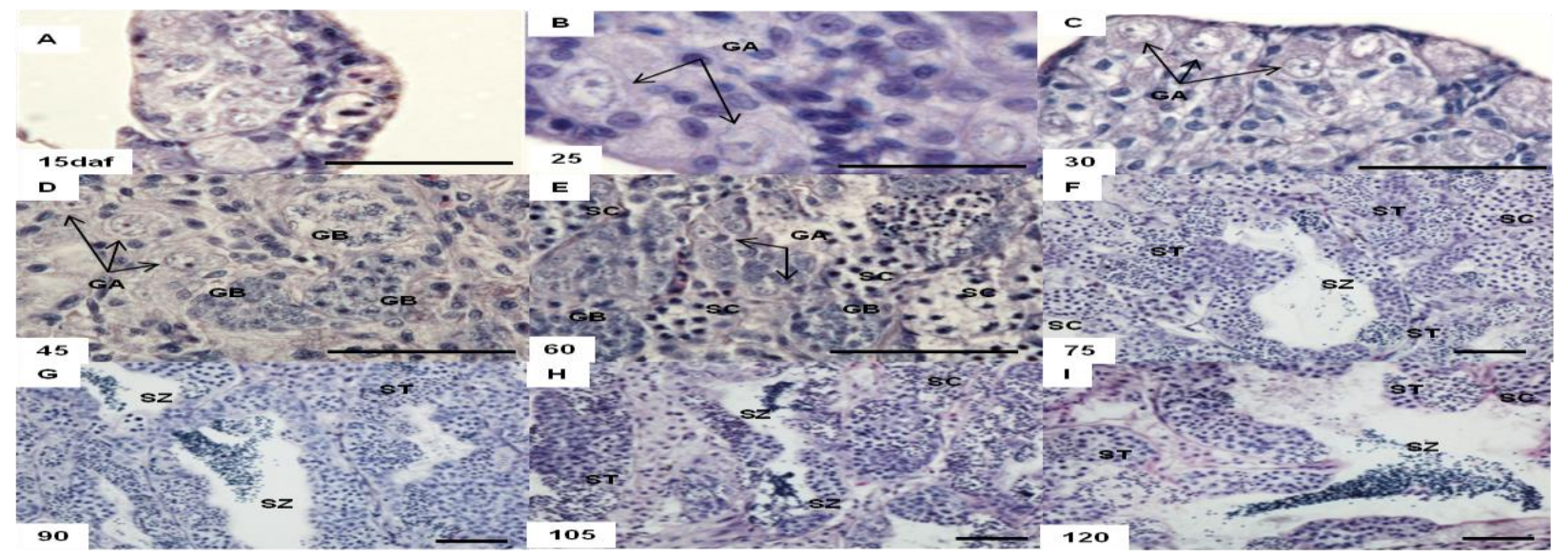

Fig.2 Microphotographs of histological sections of tilapia testes at different stages of testicular development from 15 -120 daf, all sections were stained with hematoxyline-eosin. (A) Sexual undifferentiated testicular fragments, (B) Very early type A spermatogonia, (C) Type A spermatogenia, (D) Spermatogonia A \& B type, (E) Initiation of meiosis or spermatocytes, (F) All forms of spermatogenesis, $(\mathrm{G}, \mathrm{H}, \mathrm{I})$ sexually matured stages. GA, type A spermatogonia; $\mathrm{SB}$, type B spermatogonia; SC, spermatocytes; ST, spermatids; SZ, spermatozoa (Scale bars: $50 \mu \mathrm{m}$ ). 


\section{Oocyte development during cortical alveolar to previtellogenic stage is associated with} growth rate and body weight

During oogenesis, undifferentiated oogonia containing non-dividing PGCs (Fig. 3A) around 15 daf. Morphological appearances of the nuclear envelope and nucleoli make numerous changes during oocyte growth. During 15-25 daf proliferated oogonia entered into meiotic cell division at the diplotene stage and accumulate yolk protein precursors during meiotic arrest. The oocyte at the beginning of primarily growth phase, the chromatin nucleus stages which consists of strongly basophilic cytoplasm, centrally nucleus and enlargement of oocytes gradually around 25 daf (Fig. 3B). We have observed the perinucleolar stages around 30-45 daf (Fig. 3C, D), and accumulation of cortical alveolar stages at 60 daf (Fig. 3E). Further along, pervitellogenic oocytes were observed around 75 daf (Fig. 3F). During secondary growth phase, ovarian development in the tilapia is asynchronous and oocytes of different developmental stages are simultaneously found in the matured ovary. We observed the fully grown Vtg oocyte around 90120 daf (Fig. 3G, H, I). Histological data indicate that growth rate decreased during yolk synthesis at primary growth phase of previtellogenic stages (compare Fig. 1C vs. 3F).

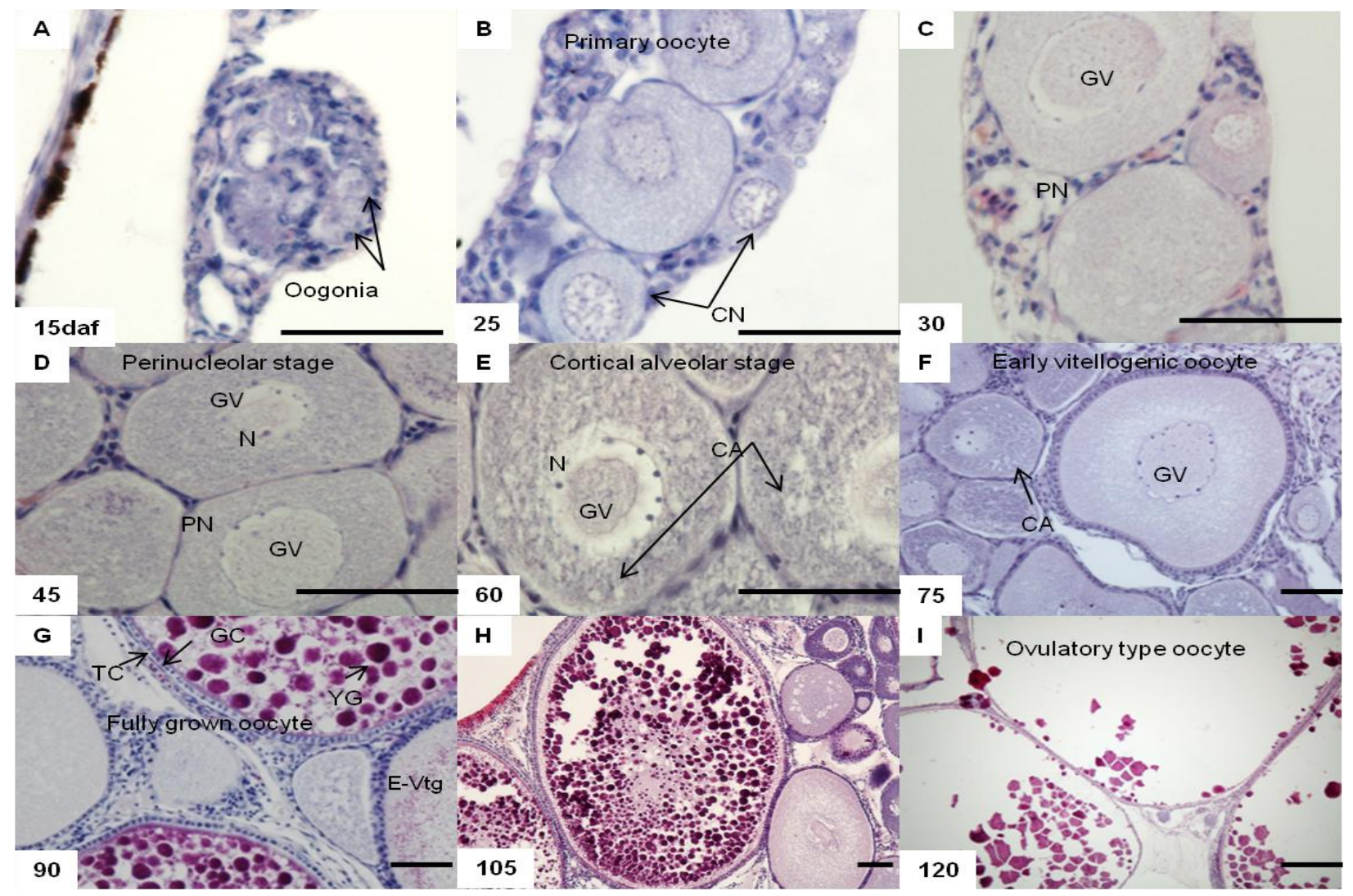

Fig.3 Microphotographs of histological sections of tilapia ovary at different stages of ovarian follicles, all sections were stained with hematoxyline-eosin. (A) Sexual undifferentiated stages, (B) Chromatin nucleolus (CN) and peri-nucleolus stages (PN), (C-D) Perinucleolar stage (PN), (E) Cortical alveolar (CA) stage, (F) Early vitellogenic (E-VTGs) oocyte, (G,H,I) Fully developing vitellogenic (VTGs) oocyte. GV, germinal vesicles; GC, granulose cells; YG, yolk globules; TC, Theca cells; N, Nucleolus (Scale bars: $50 \mu \mathrm{m}$ ). 


\section{DISCUSSION}

The goal of this study was to compare growth and reproductive axis between male and female tilapia. Interaction between growth and reproduction occurs in many vertebrates and is particularly obvious at certain stages of the life cycle [12, 13]. The present study focused on why male tilapia grow bigger than female and how growth rate is related to gonadal development between both sexes. Growth patterns of tilapia change between male and female with increasing age and sexual maturation. Male started to grow faster than female at 90 daf during the initiation of spermiogenesis when we observed the maximum growth rate at that period and decreased growth rate after final sexual maturation during spermiation. In this study, the degree of advancement of spermatogenesis during initiation of spermatogenesis to spermiogenesis was related to body weight and growth rate. This finding further confirms that growth rate was decreased after complete sexual maturation, and that sexual maturity is a major component of the determinant factors of growth in tilapia. The GSI was very low during immature stages and reached the maximum value during the mature spermatogenic stages. This suggests that at immature stages growth rate is faster compared to final stages of maturation, after spermiogenesis growth rate is very low. We conclude that the time period during spermiogenesis is very critical and interesting because during spermiogenesis male tilapia growth, growth rate and testicular maturation were positively correlated, but thereafter growth rate was exceedingly decreased (compare Fig. 1 vs.2) in male tilapia.

The major developmental events that broadly classify oogenesis have been previously reported $[7,14,15,16,17,18,19]$. It is clear that final body size of female tilapia is comparatively smaller than male (Fig. 1A, B). We provide the first physiological evidence to support the contention that, the degree of advancement of follicular development during cortical alveolar stages was related to body weight and growth rate. It is clear that oocyte growth at previtellogenesis stage affect body growth in tilapia. Thus, the early previtellogenic growth period is central in determining the age of maturation in tilapia (compare Fig. 1A, D vs. 3E, F). Therefore, the present studies clearly suggest that the initiation of previtellogenic growth period is important for growth rate. During the subsequent period ovary mass increased significantly. We observed a stepwise regression of growth rate during oocyte growth but did not observe any progressive mood of the growth rate recovery after complete sexual maturation in both sexes. However, we began to observe the mature (vitellogenic) oocytes when growth rate was rapidly decreasing. During vitellogenesis female tilapia accumulates and/or consumes enough energy to start the maturation. Furthermore, female became smaller than male in overall size and weight likely due to the slowing of growth rate in females during final gonadal development. On the other hand, the tilapias are asynchronous ovulators with their oocytes developing asynchronously and spawning frequently approximately every 2-3 weeks. Prior to and /or during these spawning periods feed intake will be markedly reduced or stop completely [20]. Somatic growth is strongly affected by maturation in Atlantic halibut [21, 22]. Research findings are limited but do suggest differences between male and female tilapia in reproductive development effecting growth rate. In tilapia, we identify two critical periods during which growth is effected during reproductive development; after spermiogenesis in males and during previtellogenic ovarian development in female. These differences allow males mature faster than females. Growth rate of females decreased earlier, resulting in overall smaller size than males and allowing males to grow faster and bigger than females. Different relationships between body size and reproductive function in 
male and females are also the predicted reasons for sexually dimorphic growth. Therefore sexual maturity is a major problem in tilapia culture due to negative effects on growth performance. Accordingly, females mature later and at a much smaller size compared to males.

In conclusion, these results demonstrate that body size growth and gonadal development are interconnected. Pubertal growth, growth rate and sexual maturation have correlated in different phase of development however this growth process was influenced by increasing sexual maturation. Male growth rate decreased after complete sexual maturation during spermiogenesis and female decreased before final sexual maturation during previtellogenic stages. Therefore, within these broad differences, there are interesting genetic variation in distribution of growth and reproduction between male and female. Female growth rate decreased earlier than male, suggesting that increasing sexual maturation inhibited size and growth rate of female.

\section{ACKNOWLEDGEMENT}

We thank Dr. Richard Hanna, La Jolla Institute for Allergy and Immunology, USA, for critical reading this manuscript. This work was supported by grant from Japan Society for the Promotion of Science (JSPS).

\section{REFERENCES}

[1] Fairbair D-J, Allometry For Sexual Size Dimorphism: Pattern and Process in the Coevolution of Body Size in Males and Female. Annual Review of Ecology and Systematics, 28 (1997) 659-687.

[2] Pudney J, Spermatogenesis in nonmammalian vertebrates, Microscopy Research and Technique, 32 (1995) 459-497.

[3] Loir M, Spermatogonia of rainbow trout: I Morphological characterization, mitotic activity and survival in primary cultures of testicular cells, Molecular Reproduction and Development, 53 (1999) 422-433.

[4] Miura T \& Miura C, Japanese eel: a model for analysis of spermatogenesis, Zoological Society of Japan, 18 (2001) 1055-1063.

[5] Miura T, Yamauchi K, Takahashi H \& Nagahama Y, Hormonal induction of all stages of spermatogenesis in vitro in the male Japanese eel (Anguilla japonica), Proceeding of National Academic Sciences, 88 (1991) 5774-5778.

[6] Schulz R W, Franca L R, Lareyre J J, Le Gac F, Chiarini-Garcia H, Nobrega R \& Miura T, Spermatogenesis in fish, General and Comparative Endocrinology, 165 (2010) 390411.

[7] Tyler C R \& Sumpter J R, Oocyte growth and development in teleosts, Reviews in fish Biology and Fisheries, 6 (1996) 287-318.

[8] Miura C, Higashino T \& Miura T, A Progestin and an Estrogen Regulate Early Stages of Oogenesis in fish, Biology of Reproduction, 77 (2007) 822-828. 
[9] Nagahama Y, Endocrine regulation of gametogenesis in fish, International Journal of Developmental Biology, 38 (1994) 217-229.

[10] Selman K, Wallace R A \& Barr V, Oogenesis in Fundulus heteroclitus. IV. Yolk vesicle formation, Journal of Experimental Zoology, 129 (1986) 277-88.

[11] Selman K, Wallace R A, Sarka A, \& Xiaoping Q, Stages of oocyte development in the zebrafish, Brachydanio rerio, Journal of Morphology, 218 (1993) 203-24.

[12] Campbell B, Dickey J, Beckman B, Young G, Pierce A \& Swanson P, Endocrine changes associated with the growth of pre-vitellogenic oocytes in coho salmon (Oncorhynchus kisutch), Fish Physiology and Biochemistry, 28 (2003) 287-289.

[13] Campbell B, Dickey J, Beckman B \& Young G, Previtellogenic oocyte growth in Salmon: relationship among body growth Plasma insulin-like growth factor-I, estradiol$17 \beta$, follicle -stimulating hormone and expression of ovarian genes for insulin-like growth factors, steroidogenic-acute regulatory protein and receptors for gonadotropins, growth hormone and somatolactin, Biology of Reproduction, 75 (2006) 34-44.

[14] Nahamura M \& Nagahama Y, Ultrastructural study on the differentiation and development of steroid-producing cells during ovarian differentiation in the Amago salmon, Oncorhynehus rhodurus, Aquaculture, 112 (237) (1993) 51.

[15] Selman K \& Wallace R A, Oogenesis in Fundulus heteroclitus. III. Vitellogenesis, Journal of Experimental Zoology, 226 (1993) 441-457.

[16] Nagahama Y, 17 $\alpha, 20 \beta$-Dihydroxy-4-pregnen-3-one: A teleost maturation-inducing hormone, Development, Growth \& Differentiation, 29 (1987b) 1-12.

[17] Nagahama Y, Endocrine control of oocyte maturation. In Hormones and Reproduction in Fishes, Amphibians, and Reptiles. pp. 171-202, Edited by Norris D O and Jones R E, Plenum Press, New York, (1987c).

[18] Nagahama Y, Michiyasu Yoshikuni, Masakane Yamashita, Noriyoshi Sakai and Minoru Tanaka, Molecular endocrinology of oocyte growth and maturation in fish, Fish Physiology and Biochemistry, 3 (1993) 14.

[19] Goetz F W, Hormonal control of oocyte final maturation and ovulation in fishes, In Fish Physiology, Vol. IXA, pp. 117-170, Edited by Hoar W S, Randall D J and Donaldson E M, Academic Press, New York, (1983).

[20] Kadri S, Mitchell D F, Metcalfe N B, Huntingford F A \& Thorpe J E, Differential patterns of feeding and resource accumulation in maturing and immature Atlantic salmon, Salmo salar Aquaculture, 142 (1996) 245-257. 
[21] Norberg B, Weltzien F A, Karlsen O \& Holm J C, Effects of photoperiod on sexual maturation and somatic growth in male Atlantic halibut (Hippoglossus hippoglossus L.), Comparative Biochemistry and. Physiology, 129B (2001) 357-365.

[22] Weltzien F A, Karlsen O \& Norberg B, Growth patterns and plasma levels of testosterone, 11- ketotestosterone, and IGF-1 in male Atlantic halibut (Hippoglossus hippoglossus) from juvenile stages throughout sexual development, Fish Physiology and Biochemistry, 28 (1-4) (2003a) 227-228. 